\title{
A proposed Italian contribution to the MIRAX Scientific Payload
}

L. Amati $\left({ }^{1}\right)$, M. Feroci $\left({ }^{2}\right)$, F. Frontera $\left({ }^{3}\right)$, C. $\operatorname{Labanti}\left({ }^{1}\right), \operatorname{A.} \operatorname{Vacchi}\left({ }^{4}\right)$, A. Argan $\left({ }^{5}\right)$, R. Campana $\left({ }^{2}\right)$, E. $\operatorname{Costa}\left({ }^{2}\right)$, R. Ruffini $\left({ }^{6}\right)$, I. Bombaci $\left({ }^{7}\right)$, E. Del Monte $\left({ }^{2}\right)$, I. Donnarumma $\left({ }^{2}\right)$, A. $\operatorname{Drago}\left({ }^{3}\right)$, Y. Evangelista $\left({ }^{2}\right)$, R. Farineldi $\left({ }^{3}\right)$, G. Ghirlanda $\left({ }^{8}\right)$, G. Ghisellini $\left({ }^{8}\right)$, C. Guidorzi $\left({ }^{3}\right)$, F. Fuschino $\left({ }^{1}\right)$, F. Lazzarotto $\left({ }^{2}\right)$, D. Lazzati $\left({ }^{9}\right)$, P. Malcovati $\left({ }^{10}\right)$, M. Marisaldi $\left({ }^{1}\right)$, E. Moreldi $\left({ }^{1}\right)$, F. Muleri $\left({ }^{2}\right)$, M. Orlandini $\left({ }^{1}\right)$, L. $\operatorname{Pacciani}\left({ }^{2}\right)$, E. Pian $\left({ }^{11}\right)$, M. Rapisarda $\left({ }^{2}\right)$, A. Rubini $\left({ }^{2}\right)$, R. Salvaterra $\left({ }^{12}\right), \operatorname{P} . \operatorname{Soffitta}\left({ }^{2}\right)$, L. Titarchuk $\left({ }^{3}\right)$, A. Traci $\left({ }^{1}\right)$, A. Rashevsky $\left({ }^{4}\right)$, G. Zampa $\left({ }^{4}\right)$, N. Zampa $\left({ }^{4}\right)$, N. Auricchio $\left({ }^{1}\right)$, A. Basili $\left({ }^{1}\right)$, E. $\operatorname{Caroli}\left({ }^{1}\right)$, E. Maiorano $\left({ }^{1}\right)$, N. Masetti $\left({ }^{1}\right)$, L. $\operatorname{Nicastro}\left({ }^{1}\right)$, E. Palazzi $\left({ }^{1}\right)$, S. Silvestri $\left({ }^{1}\right)$, J. B. $\operatorname{Stephen}\left({ }^{1}\right)$ and J. Braga $\left({ }^{13}\right)$

$\left({ }^{1}\right)$ INAF, Istituto di Astrofisica Spaziale e Fisica Cosmica - Bologna, Italy

$\left(^{2}\right)$ INAF, Istituto di Astrofisica Spaziale e Fisica Cosmica - Rome, Italy

$\left({ }^{3}\right)$ Università di Ferrara - Ferrara, Italy

$\left.{ }^{4}\right)$ INFN, Sezione di Trieste - Trieste, Italy

$\left({ }^{5}\right)$ INAF, sede centrale - Rome, Italy

$\left({ }^{6}\right)$ International Center for Relativistic Astrophysics Network (ICRANet) - Pescara, Italy

$\left(^{7}\right)$ Università di Pisa - Pisa, Italy

$\left.{ }^{8}\right)$ INAF, Osservatorio Astronomico di Brera - Merate, Italy

$\left({ }^{9}\right)$ North Carolina State University - Raleigh, NC, USA

$\left({ }^{10}\right)$ Università di Pavia - Pavia, Italy

$\left.{ }^{11}\right)$ INAF, Osservatorio Astronomico di Trieste - Trieste, Italy

$\left.{ }^{(12}\right)$ Università dell'Insubria - Como, Italy

$\left({ }^{13}\right)$ Instituto Nacional de Pesquisas Espaciais (INPE) - Sao Josè dos Campos, Brazil

(ricevuto il 25 Febbraio 2011)

Summary. - We describe the mission opportunity, the science case, the instrument concept and the preliminary expected performances of the GRB/all-sky X-ray monitor proposed by an Italian collaboration for the inclusion in the scientific payload of the MIRAX X-ray astrophysics mission, which will fly in 2015 on board the Brazilian satellite Lattes.

PACS 95.55.Ka - X- and $\gamma$-ray telescopes and instrumentation.

PACS 98.70.Qy - X-ray sources; X-ray bursts.

PACS 98.70.Rz - $\gamma$-ray sources; $\gamma$-ray bursts. 


\section{1. - The MIRAX mission opportunity}

MIRAX is the first Brazilian astrophysical space mission (INPE; P.I Prof. J. Braga), scheduled for a flght in a nearly equatorial low Earth $(\sim 600 \mathrm{~km})$ orbit on board the LATTES satellite in the 2015-2016 timeline. In addition to MIRAX, LATTES will carry also the geophysical mission EQUARS, exploiting to the multi-payload platform (PMM) developed by INPE. The mass and power budget available for the MIRAX payload are $120 \mathrm{~kg}$ and $90 \mathrm{~W}$, respectively; the data link will likely be through the $X$ band. In its present configuration, MIRAX consists of a small CZT coded mask camera (Hard X-ray Imager, HXI) of limited area, FOV and spectral capabilities, with the primary scientific objective of monitoring the Galactic center in the 10-200 keV energy band [1].

Following contacts and meetings with Prof. Joao Braga, from the INPE (Brazil) and PI of the MIRAX mission, of an Italian collaboration including INAF/IASF Bologna, INAF/IASF Rome, University of Ferrara, INFN Trieste, and ICRAnet, a payload proposal has been submitted and discussed with the INPE, in a meeting held in June 2010. The instrumentation proposed is expected to give a high scientific return in the field of the GRB astrophysics and in sky monitoring of celestial sources. It exploits: a) the R\&D activities supported in the last years by ASI, INAF and INFN for innovative Silicon Drift Detectors; b) the experience acquired from the PI-ship of the BeppoSAX PDS and GRBM instruments; c) the experience acquired from the PI-ship of the Super-AGILE experiment; d) the excellent competence acquired in the data analysis and interpretation of the GRB and celestial source observations.

\section{2. - Scientific goals of the Italian proposed payload}

The MIRAX payload proposed by the Italian collaboration has two main scientific objectives:

a) performing spectral and timing measurements of the prompt emission of GammaRay Bursts (GRBs) over a broad energy band, from $\sim 1 \mathrm{keV}$ to $10 \mathrm{MeV}$, combined with location accuracy of a few arcmin;

b) monitoring celestial X-ray sources in $2-50 \mathrm{keV}$ with a broad FOV, a few arcmin source location accuracy and a few mCrab daily sensitivity.

$2 \cdot 1$. Gamma-ray bursts. - Despite the huge observational efforts of the last decades in the study of the GRB phenomenon, which provided a good characterization of the bursts temporal and spectral properties, the accurate localization and consequent discovery of their multi-wavelength afterglow emission, the determination of their cosmological distance scale and the evidence of a connection with peculiar type $\mathrm{Ib} / \mathrm{c} \mathrm{SNe}$, several relevant open issues have still to be addressed, both from the observational and theoretical points of view [2].

Under this respect, there is a general consensus that the study of the prompt emission of GRBs over a broad energy band extending from several MeVs down to $\sim 1 \mathrm{keV}$ is crucial. By fulfilling these requirements, together with a source location accuracy of a few arcmin, MIRAX will address scientific goals of fundamental importance and not achieved by GRB experiments presently flying (e.g., Swift, Konus/WIND, Fermi/GBM, $\mathrm{MAXI}$ ) and future approved missions (SVOM). These can be summarized as follows:

- to perform an unbiased measurement of the GRB time-resolved spectral shape and its evolution down to about $1 \mathrm{keV}$ in photon energy, which is crucial for testing 
models of GRB prompt emission (still to be settled despite the considerable amount of observations);

- to detect and study transient X-ray absorption column/features for tens of medium/bright GRBs per year. These measurements are of paramount importance for the understanding of the properties of the Circum-Burst Matter (CBM) and hence the nature of GRB progenitors (still a fundamental open issue in the field). In addition, as demonstrated by us with BeppoSAX [3], the detection of transient features can allow the determination of the GRB redshift to be compared, when it is the case, with that determined from the optical/NIR lines;

- to provide a substantial increase (with respect to the past and current missions) in the detection rate of X-Ray Flashes (XRF), a sub-class of soft/ultra-soft events which could constitute the bulk of the GRB population and still have to be explored satisfactorily;

- to extend the GRB detection up to very high redshift $(z>8)$ GRBs, which is of fundamental importance for the study of evolutionary effects, the tracing of star formation rate, ISM evolution, and possible unveiling of population III stars;

- to perform an accurate determination of spectral peak energy, which is a fundamental quantity for the test and study of spectrum-energy correlations and the possible use of GRBs as cosmological probes;

- to study the erratic time variability down to sub-millisecond time scale. This is crucial to establish the intrinsic Magneto-Hydrodynamic (MHD) time scales of the GRB source;

- to achieve a good detection efficiency for short/hard GRBs, whose difference with long GRB in terms of progenitors and emission physics is a central issue in the field;

- to provide fast and accurate location of the detected GRBs to allow their prompt multi-wavelength follow-up with ground and space telescopes, thus leading to the identification of the optical counterparts and/or host galaxies and to estimate their redshift, a fundamental measurement for the scientific goals listed above, comparing it with that determined from X-ray absorption lines (see above).

2 2. X-ray all-sky monitoring. - The most sensitive observatories have in general a narrow field of view ( $\sim 1 \mathrm{deg}$ or less) and are designed to perform studies of individual sources. However also a wide field instrumentation is needed. One of its goals is to trigger Target of Opportunity Observations (TOO) with the narrow field observatories, to catch the most interesting states of the sources, often unpredictable, with very high sensitivities. Also, deep observations of persistent but variable sources are requested to explore the history or evolution of the source (or class of sources). Indeed, often wide field telescopes have been part of the payload of many satellite X-ray missions (e.g., RXTE, BeppoSAX, INTEGRAL, Swift, AGILE, Suzaku) and are desired to be also in future missions. Indeed, the most recent ASTRONET report (The ASTRONET Roadmap: A Strategic Plan for European Astronomy) identified the All Sky Monitoring as one of two specific gaps in the strategic planning for the future European space missions.

Independently of the service function, long-term, continuous monitoring of celestial $\mathrm{X}$-ray sources allows the study of source/class properties not easily or inaccessible to 
specific, short observations, although more sensitive. Examples include the discovery of new Galactic transient sources, the discovery and long-term evolution of orbital, superorbital and spin periodicities, period derivatives and quasi-periodicities (QPOs), the intensity and spectral state changes in BHC, the multi-frequency correlation between timing, spectral and intensity parameters, the discovery and monitoring of bursting behaviour (bursters, SGRs, AXPs, ...), complete all sky surveys. In addition to Galactic sources, All Sky Monitoring is also important for AGN studies, as shown, e.g., by the recent Swift/BAT survey [4]. The real need is for a simultaneous monitoring of the largest fraction of the sky. In fact, it is worth stressing the fundamental difference between "sampling" and "continuous monitoring" of the sky. The former is much less demanding in terms of instrumental resources, both for hardware and mission operation. However, when the target sources are transient and variable on short timescales, the difference between short and sparse observations and continuous monitoring may be crucial.

Summarizing, our scientific objectives for all-sky monitoring include:

- detection and localization within a few arcimn of Soft Gamma Repeaters (SGR) and many other classes of galactic X-Ray Transients (XRT), like, e.g., galactic low- and high-mass X-ray binaries in outburst, cataclismic variables, accreting ms pulsars, etc., for spectral and timing studies and to trigger follow-up observations by ground and space observatories (included SRG itself), a fundamental service for the world-wide community, given the expected demise of RXTE/ASM in the next few years;

- to perform an all-sky survey in the 1-50 keV complementary, e.g., to that by eROSITA at lower energies.

\section{3. - Instrument concept}

The Italian payload we propose for MIRAX is composed of two independent instruments, aimed at the two main observational objectives: a) detection, localization and wide-band (about $1 \mathrm{keV}-10 \mathrm{MeV}$ ) spectral and timing measurements of Gamma Ray Bursts (GRBs); b) All Sky Monitoring, i.e. long-term monitoring of celestial sources and discovery of new ones. Particular care will be devoted to the monitoring of the Galactic plane. The main requirements are thus a broad energy band extending from several MeVs down to $\sim 1 \mathrm{keV}$, an energy resolution of a few hundreds of $\mathrm{eV}$ at energies $<20 \mathrm{keV}$, a source location accuracy of a few arcmin and a Field of View (FOV) of several sr. The instrumentation is then composed of thee pairs of coded-mask X-ray imagers (X-Ray Monitor, XRM) and two modules of 4 scintillator spectrometer units (Soft Gamma-ray Spectrometer, SGS). The instrumental approach to achieve the science goals is to use separate detectors for the low and high part of the energy band.

The low energy (about 1-50 keV) is best covered with silicon detectors, whereas the high energy portion of the spectrum $(15-10000 \mathrm{keV})$ is covered with inorganic scintillators in phoswich configuration. Given that the LATTES satellite hosts along with MIRAX the payload EQUARS devoted to the observation of the Earth, it will move like the International Space Station, with the MIRAX zenith continuously drifting and covering $360 \mathrm{deg}$ in each orbit. This zenith-drift properties allows to observe almost the entire sky in an orbit if the field of view of the MIRAX telescopes is as large as possible in the direction orthogonal to the direction of motion of the spacecraft, while in the other direction it can be smaller, being covered through the satellite motion. 

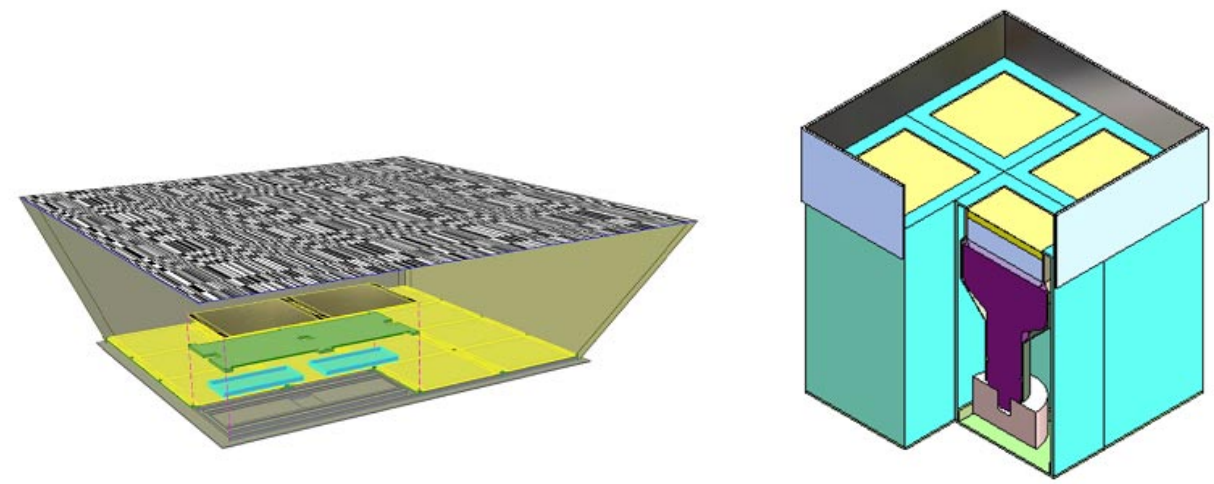

Fig. 1. - Pictorial views of an XRM unit (left) and an SGS module (right).

We have achieved this goal by adopting the configuration illustrated in figs. 1 and 2 . The XRM consists of a 3 pairs of Silicon Drift detectors (initially developed for the ALICE experiment for the CERN/LHC accelerator) surmounted by mono-dimensional coded masks [5]. The SGS consists of two modules of 4 phoswich (PHOSsphor sandWICH) units each. The detector units are made of $\mathrm{NaI}(\mathrm{Tl})$ and $\mathrm{CsI}(\mathrm{Na})$ scintillators viewed from a single photomultiplier (PMT). The photon energy lost in each scintillator can be established from the pulse shape analysis of the signals provided by the PMT. The same technique was successfully developed for the PDS instrument aboard BeppoSAX, one of the most sensitive instruments launched thus far. The phoswich configuration is the best technique to derive unbiased spectra in the hard X/gamma-ray energy band [6].

\section{4. - Expected performances}

Table I summarizes the expected main characteristics of the XRM and SGS instruments. Based on these features, we evaluated the expected performances of our proposed payload both for the GRB and ASM science. In these simulations we took into account
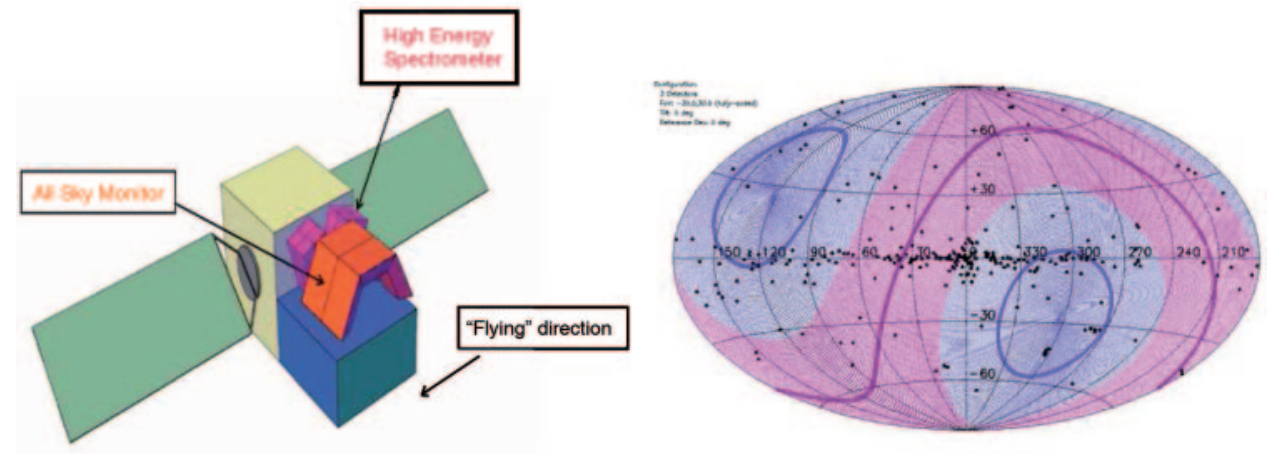

Fig. 2. - Left: a hypothesis of allocation of the XRM and SGS experiments onboard the Lattes satellite. Right: the sky accessible to the ASM experiment after every orbit (90 minutes). Thick lines represent the center of the field of view of the three subsystems. 
TABLE I. - Main characteristics of the proposed MIRAX payload.

\begin{tabular}{lll}
\hline & XRM & SGS \\
\hline Energy band $(\mathrm{keV})$ & $2-50$ & $15-10000$ \\
Energy resolution $(\mathrm{keV})$ & 0.25 at $6 \mathrm{keV}$ & 10 at $60 \mathrm{keV}$ \\
& 0.5 at $50 \mathrm{keV}$ & 50 at $662 \mathrm{keV}$ \\
Timing resolution $(\mathrm{ms})$ & 0.01 & 0.001 \\
Field of view $(\mathrm{sr})$ & $2.5\left(^{a}\right), 3.5\left(^{b}\right)$ & 3.0 \\
Effective area $\left(\mathrm{cm}^{2}\right)$ & $\sim 550\left(\left(^{c}\right)\right.$ & $\sim 700-1000$ \\
Angular resolution $\left(^{\prime}\right)$ & $\sim 5$ & - \\
Source location accuracy $\left(^{\prime}\right)$ & $\sim 1$ & $\sim 1(1 \mathrm{~s})$ \\
Sensitivity $(\mathrm{mCrab})\left({ }^{d}\right)$ & $700(1 \mathrm{~s})$ & \\
& $90(60 \mathrm{~s})$ & \\
& $<26(1 \mathrm{orbit}$, half sky) & $\sim 40$ \\
\hline
\end{tabular}

$\left(\begin{array}{l}a \\ { }^{b}\end{array}\right)$ Fully coded.

$\left({ }^{b}\right)$ Partially coded.

$\left({ }^{c}\right)$ In the fully coded FOV.

$\left(^{d}\right)$ The reported sensitivity is at $5 \sigma$ significance level. For the XRM we report the sensitivity for 1 detector in imaging mode for the fully coded FOV; for the spectroscopy mode, the sensitivity of the XRM is expected to be a factor of $\sim 1.4$ better.

the expected orbit and inclination of the LATTES satellite, we adopted the standard level and spectrum of the diffuse cosmic X-ray background and we based on previous experiments (BeppoSAX, superAGILE) for the evaluation of the "intrinsic" background.

In fig. 2 (right panel), it is shown the MIRAX payload capability of surveying the almost entire sky per orbit. The effective area of each of the two MIRAX Italian instruments, XRM and SGS, for any given sky direction, is shown in the left panel of fig. 3. As an example of simulation of the spectral MIRAX/XRM+SGS capabilities, in

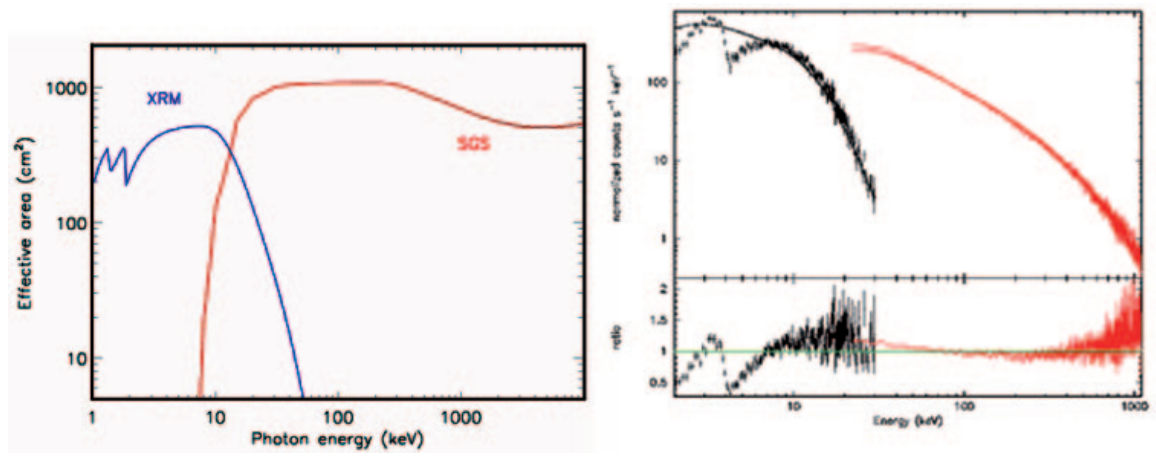

Fig. 3. - Left: effective area as a function of energy of the XRM (2 modules) and for the SGS instrument (2 modules). Right: the spectrum of the first $13 \mathrm{~s}$ of GRB 990705 characterized by a transient absorption edge at $3.8 \mathrm{keV}$ [3] as would be measured by the XRM (left) and SGS (right). The residuals of the fit with a Band spectral model with no edge are shown. 
the right panel of fig. 3 we show the transient absorption edge at $3.8 \mathrm{keV}$ detected with BeppoSAX/WFC from GRB990705 [3] as it would be detected with MIRAX. The much better capability of MIRAX is outstanding: the same line observed with WFC at a significance level of about 5 sigma is expected to be detected with MIRAX at a significance level of 50 sigma. This means that with MIRAX we expect to detect transient absorption lines about one order of magnitude weaker.

\section{REFERENCES}

[1] Braga J. and Mejía J., Proc. SPIE, 6266 (2006) 17.

[2] Gehrels N., Ramirez-Ruiz E. and Fox D. B., Annu. Rev. Astron. Astrophys., 47 (2009) 567.

[3] Amati L., Frontera F., Vietri L. et al., Science, 290 (2000) 953.

[4] Ajello M., Costamante L., Sambruna R. et al., Astrophys. J., 699 (2009) 603.

[5] Feroci M., Amati L., Antonelli L. A. et al., AiP Conf. Proc., 1133 (2009) 49.

[6] Frontera F., Costa E., Dal Fiume D. et al., Astron. Astrophys. Suppl., 122 (1997) 357. 